\title{
Biliary ascariasis: particular cause of biliary tract infection
}

\author{
Yanting Wang, Jinheng Liu \\ Department of Hepatobiliary-Pancreatic Surgery, Chengdu Second People's Hospital, Chengdu 610017, China \\ Correspondence to: Jinheng Liu, MD. Department of Hepatobiliary-Pancreatic Surgery, Chengdu Second People's Hospital, No.10 Qingyun South \\ Street, Chengdu 610017, China. Email: doctorliujinheng@sina.com.
}

Submitted Oct 08, 2019. Accepted for publication Oct 24, 2019.

doi: 10.21037/hbsn.2019.11.01

View this article at: http://dx.doi.org/10.21037/hbsn.2019.11.01

A 57-year-old previously healthy woman manifested a 6-day history of severe epigastric pain along with nausea and vomiting. There was tenderness in the epigastrium, without rebound pain or guarding. Laboratory examinations, including white blood cell count, neutrophilic granulocyte percentage, glutamic-pyruvic transaminase, aspartate aminotransferase, were rise. Computed tomography (CT) of the abdomen showed mild dilatation of the common bile duct that a strip-type high-density shadow was in it without enhancement (Figure 1A,B). Magnetic resonance cholangiopancreatography (MRCP) showed a striptype low-signal shadow was in the common bile duct and the right hepatic duct (Figure 1C). The Biliary ascariasis was diagnosed based on the patient's clinical history and image features. The Ascaris was removed with the use of endoscopic forceps, and another Ascaris could be seen in the duodenum during surgery (Figure 1D). Pathological examination identified as Ascaris. The patient was given
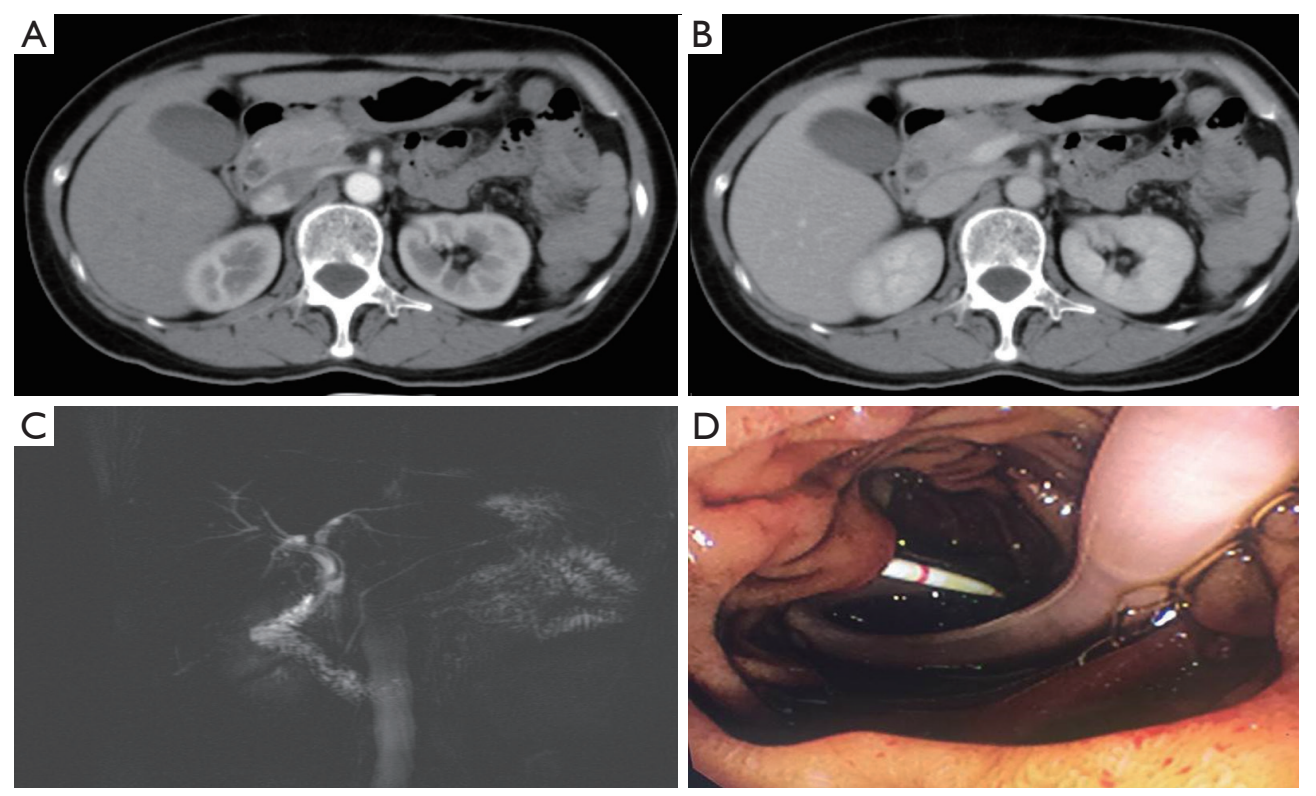

Figure 1 Images of Ascaris. (A) A strip-type high-density shadow in the common bile duct; (B) without enhancement in the venous phase; (C) a strip-type low signal shadow in the common bile duct and the right hepatic duct; (D) Ascaris in the duodenum. 
albendazole to prevent recurrence after surgery.

\section{Acknowledgments}

The authors thank Professor Zhang Gang for providing help to this research.

\section{Footnote}

Conflicts of Interest: The authors have no conflicts of interest to declare.
Ethical Statement: The authors are accountable for all aspects of the work in ensuring that questions related to the accuracy or integrity of any part of the work are appropriately investigated and resolved. Written informed consent was obtained from the patient for publication of this manuscript and any accompanying images.

Cite this article as: Wang Y, Liu J. Biliary ascariasis: particular cause of biliary tract infection. Hepatobiliary Surg Nutr 2020;9(1):119-120. doi: 10.21037/hbsn.2019.11.01 